\title{
Atributos essenciais da Atenção Primária à Saúde: resultados nacionais do PMAQ-AB
}

\author{
Essential attributes of Primary Health Care: national results of \\ $P M A Q-A B$
}

Juliana Gagno Lima', Ligia Giovanella², Márcia Cristina Rodrigues Fausto³, Aylene Bousquat ${ }^{\mathbf{4}}$, Edcarlos Vasconcelos da Silva ${ }^{5}$

DOI: 10.1590/0103-11042018S104

\footnotetext{
1 Universidade Federal do Oeste do Pará (Ufopa), Instituto de Saúde Coletiva (Isco) - Santarém (PA) Brasil.

RESUMO A concepção de uma Atenção Primária à Saúde (APS) abrangente e sua qualidade podem ser avaliadas por meio da efetivação dos atributos: primeiro contato, longitudinalidade, abrangência/integralidade e coordenação. $\mathrm{O}$ artigo teve por objetivo analisar a consecução dos atributos da APS na prática das equipes, na perspectiva dos profissionais e usuários, comparando-se os resultados do Programa Nacional de Melhoria do Acesso e da Qualidade da Atenção Básica (PMAQ-AB), entre os ciclos 1 e 2. Estudo quantitativo, com identificação das equipes participantes dos dois ciclos e operacionalização dos atributos da APS por meio da seleção de variáveis PMAQ-AB pertinentes a cada atributo. Foram comparados os resultados nacionais de 12.680 Unidades Básicas de Saúde (UBS), 15.668 equipes e 58.517 usuários. Apenas parte dos atributos apresentou melhorias do ciclo 1 para o ciclo 2. Os melhores resultados foram primeiro contato e integralidade, confirmando gradual constituição da UBS como serviço de porta de entrada acessível, com escopo diversificado de ações, ainda que insuficientes. Os atributos com pior avaliação foram longitudinalidade, com baixa continuidade e qualidade da relação profissional-paciente; e coordenação, por dificuldades de acesso às consultas especializadas e insuficientes registros compartilhados.

PALAVRAS-CHAVE Atenção Primária à Saúde. Acesso. Integralidade.

ABSTRACT The concept of a comprehensive Primary Health Care (PHC) and its quality can be evaluated by the effectiveness of the following attributes: first contact service, longitudinality, comprehensiveness and coordination. The objective of this article is to analyze the effectiveness of PHC attributes in team practice from the perspective of professionals and users, comparing the results of the National Program for Access and Quality Improvement in Primary Health Care (PMAQ-AB), between cycles 1 and 2. Quantitative study, with identification of the teams participating in the two cycles and operationalization of the PHS attributes through the selection of $P M A Q-A B$ variables relevant to each attribute. We compared the national results of 12.680 Basic Health Units, 15.668 teams and 58.517 users. Some attributes improved from cycle 1 to cycle 2. The best results were first contact and comprehensiveness, confirming the gradual constitution of the Basic Health Units as an accessible first contact service, with a diversified scope of actions, although insufficient. The attributes with worse evaluation were longitudinality, with low continuity and quality of the professional-patient relationship; and coordination, due to difficulties of access to specialized care and insufficient shared records.

KEYWORDS Primary Health Care. Accessibility. Comprehensiveness. 


\section{Introdução}

Nas últimas décadas, a Atenção Primária à Saúde (APS) tem sido revitalizada, haja vista sua centralidade para a melhoria dos cuidados e dos resultados em saúde. Estudos internacionais apontam que a APS forte e articulada a outros níveis de atenção em sistemas universais contribui para impactos positivos na saúde da população. São eles: maior e melhor acesso aos serviços de acordo com a necessidade; maior enfoque em prevenção e promoção; redução da mortalidade; maior qualidade no atendimento; diagnóstico e tratamento precoce de doenças; redução de procedimentos especializados desnecessários e potencialmente prejudiciais $\mathbf{1}$.

A APS abrangente ou integral é fundamentada na compreensão da saúde como direito e está relacionada com os processos de desenvolvimento social e da determinação social da doença ${ }^{2}$. Um dos pontos centrais dessa perspectiva é a integração da APS ao sistema de saúde para a garantia do cuidado integral, acesso e cobertura universal com base em necessidades em saúde da população.

A concepção de APS abrangente pode ser operacionalizada com foco na discussão da qualidade da atenção, considerando quatro atributos essenciais para sua avaliação: primeiro contato, longitudinalidade, integralidade e coordenação ${ }^{3}$. No Brasil, a concepção de APS abrangente está presente na Política Nacional de Atenção Básica (PNAB) 4 .

Desde a implantação do SUS, o acesso à Atenção Básica (AB) foi expandido, sendo que a Estratégia Saúde da Família (ESF) preconizou um novo modelo assistencial para APS no País. Esse processo demandou a avaliação dos serviços ofertados à população com vistas a fornecer subsídios para a melhoria da qualidade da atenção. A indução de políticas do governo federal para a reorientação do modelo de organização da atenção foi marcada por esforços no sentido de fortalecer as estratégias de monitoramento e avaliação da $\mathrm{AB}^{5}$.
Em 2011, o Departamento de Atenção Básica do Ministério da Saúde (DAB/MS) implementou o Programa Nacional para Melhoria do Acesso e Qualidade da Atenção Básica (PMAQ-AB) ${ }^{6}$. Trata-se de um programa gerencial que tem em sua proposta a utilização da avaliação como parte do planejamento das ações para a melhoria da qualidade nos serviços de APS. Até o momento, já foram realizados dois ciclos de avaliação (2011/2012 e 2013/2014) e iniciado o terceiro ciclo avaliativo (2017/2018).

O estudo dos atributos e da qualidade da APS reforça a necessidade de qualificação dos profissionais e da organização dos serviços para a melhoria da qualidade do sistema e, consequentemente, dos resultados de saúde 3 .

$O$ PMAQ-AB não tem seus instrumentos organizados a partir de atributos da APS, como acontece no PCATool (Primary Care Assessment Tool), que consiste em um conjunto de ferramentas de pesquisa para a avaliação dos serviços de APS, adotado mundialmente e com questionário adaptado para o Brasil. Estudos com base no PCATool apontam que alto desempenho das equipes está associado com maior qualidade do cuidado para problemas de saúde de alta prevalência, como, por exemplo, as doenças crônicas 7 ,8.

Destarte, este artigo visa analisar a consecução dos atributos da APS na prática das equipes, na perspectiva dos profissionais e usuários, comparando-se os resultados do PMAQ-AB, ciclos 1 e 2 . O artigo focaliza a análise dos atributos essenciais da APS na atuação das Equipes de Saúde da Família (EqSF), observando-se modificações e permanências no instrumento ao longo dos anos de 2012 e 2014.

\section{Metodologia}

A pesquisa consiste em estudo descritivo transversal, de natureza quantitativa, baseado em dados secundários de acesso público dos ciclos 1 e 2 do PMAQ-AB 
(2011/2012 e 2013/2014 respectivamente). Os bancos de dados do ciclo 1 e ciclo 2 estão disponibilizados no site do DAB/MS.

Embora os instrumentos utilizados para a coleta de dados da avaliação externa do PMAQ-AB não estejam organizados a partir dos atributos definidos por Starfield ${ }^{\mathbf{3}}$ para a qualificação da APS, buscou-se estabelecer uma correspondência entre questões selecionadas do PMAQ-AB e os atributos de qualidade da APS descritos pela autora.

Para a seleção das variáveis do PMAQ-AB, realizou-se uma busca e seleção nos instrumentos do Programa, das variáveis correspondentes aos componentes envolvidos com cada atributo. Essa seleção foi realizada pela equipe da Pesquisa Regiões e Redes Dimensão APS (resbr.net.br). Buscou-se examinar a efetivação de cada atributo da APS de acordo com as respostas dos profissionais e dos usuários. Foram analisadas a pertinência, de acordo com a concepção de cada atributo, e uma tabulação preliminar dos bancos de dados, identificando-se a disponibilidade e a qualidade dos dados das variáveis pré-selecionadas do questionário. Entende-se variável aqui por cada resposta do instrumento utilizada, ou seja, uma mesma pergunta do instrumento pode gerar uma ou mais variáveis.

Os atributos essenciais da APS, a partir da revisão de literatura e análise de metodologias que avaliam esses atributos, foram operacionalizados em componentes considerando-se também a disponibilidade das variáveis do instrumento PMAQ-AB.

A fim de facilitar uma visualização de possíveis mudanças nos resultados entre um ciclo e outro, foram comparados os resultados do ciclo 1 e ciclo 2, utilizando-se, para tal, as mesmas Unidades Básicas de Saúde (UBS) e equipes participantes de ambos os ciclos, totalizando em comum: 12.860 UBS e 15.668 EqSF. Para análise das respostas dos usuários, foram 'selecionados' aqueles correspondentes às equipes que participaram dos dois ciclos, sendo 58.517 usuários do ciclo $1 \mathrm{e}$ 59.763 usuários do ciclo 2 das EqSF.
Para o estudo, a análise estatística foi realizada a partir do Teste de Qui-Quadrado para proporções esperadas iguais para uma amostra com variável nominal. Este consiste em um teste de aderência e é empregado para avaliar se os escores observados e os esperados (calculados) são iguais. Assim, pôde-se avaliar se há diferenças de proporções entre os que responderam 'sim' no primeiro ciclo da pesquisa PMAQ e os que responderam 'sim' no segundo ciclo com as seguintes hipóteses de teste:

- HO - As proporções entre os que responderam 'sim' do primeiro e os que responderam 'sim' no segundo ciclo do PMAQ são iguais.

- H1 - As proporções entre os que responderam 'sim' do primeiro e os que responderam 'sim' no segundo ciclo do PMAQ são diferentes e estatisticamente significativas.

Valores de p-valor abaixo de 0.05 foram considerados estatisticamente significativos. Os dados foram analisados pelo programa Bioestat versão 5.3, software free.

A partir dos resultados nacionais das variáveis selecionadas do PMAQ-AB, buscou-se identificar resultados superiores, inferiores ou mantidos sobre os atributos da APS, nos dois ciclos da avaliação externa (2012 e 2014), tendo seus resultados expostos por atributo e considerando-se apenas variáveis compatíveis entre os ciclos. O questionário do PMAQ-AB vem sendo revisado a cada ciclo, e variáveis são excluídas, reformuladas e introduzidas.

Pôde-se sintetizar os conceitos dos atributos essenciais, conforme abaixo:

- Primeiro contato: serviços de porta de entrada preferencial do sistema de saúde, procurados cada vez que os usuários necessitam de atenção para um problema de saúde, seja ele novo ou rotineiro. A APS deve cumprir um papel de serviço de procura regular e exercer função de filtro 
para acesso aos demais equipamentos da rede de atenção à saúde ${ }^{3,9}$.

- Longitudinalidade: responsabilidade da equipe pelo usuário ao longo da vida, através da qual a APS cumpre sua função de fonte regular de cuidados por meio da organização do serviço e do fortalecimento da relação entre profissional e paciente3,10.

- Abrangência/Integralidade: capacidade do serviço em reconhecer as necessidades do paciente e ofertar recursos diversificados para uma abordagem resolutiva no âmbito da AB. Envolve o elenco de ações dos serviços e se a forma como esses serviços são prestados são capazes de atender às necessidades da população ${ }^{3}$.

- Coordenação: capacidade de os prestadores de atenção primária coordenarem o uso de serviços no território e em outros níveis de atenção para resolver necessidades menos frequentes e mais complexas e garantir continuidade assistencial ${ }^{9}$. Relaciona-se com a continuidade da atenção entre profissionais e serviços da rede de atenção à saúde, ao acompanhamento dos casos clínicos por meio de ferramentas de informação e comunicação e à existência de referências/fluxos entre os serviços"1.

\section{Resultados e discussão}

A tabela 1 apresenta os indicadores PMAQ-AB de primeiro contato, nas dimensões horário de funcionamento, acolhimento e agendamento de consultas na $\mathrm{AB}$. Os resultados das variáveis referentes ao horário de funcionamento apresentaram elevação na quantidade de dias, porém com redução na quantidade de horas por dia. A implantação do acolhimento (de 80,9\% para 97,6\%) e reserva de vagas para atendimento no mesmo dia (de $64,9 \%$ para $88,1 \%$ ) tiveram resultados ampliados. Já nos indicadores sobre agendamento de consulta, o ponto positivo é que reduziu a proporção de usuários que, para marcar consulta, precisam fazer fila e pegar ficha antes da UBS abrir. Todavia, observou-se redução de UBS que realizam a marcação de consultas todos os dias da semana (de $55,2 \%$ em 2012 para 47,0\% em 2014).

Os dias e horários de funcionamento das UBS apresentaram-se adequados, assim como a implantação do acolhimento. Vale a reflexão, contudo, sobre a qualidade desse acolhimento, visto que foram identificadas importantes dificuldades na marcação de consultas na $\mathrm{AB}$, sendo necessário pegar ficha ou comparecer à UBS em horários específicos para marcação de consultas. Houve uma melhora entre os resultados do ciclo 1 e ciclo 2 no atributo primeiro contato, porém, a dificuldade de agendamento de consulta na $\mathrm{AB}$ em qualquer dia da semana se mantém como uma importante barreira organizacional de acesso.

Estudos mostram haver diferenças em relação à acessibilidade temporal nas UBS. O modelo padrão de funcionamento das UBS no horário das $8 \mathrm{~h}$ às $17 \mathrm{~h}$ aparece como frequente nos municípios de grande porte e limitante para o acesso da população de trabalhadores ${ }^{12}$.

Apesar de avanços para a ampliação do acesso dos serviços da APS, observa-se desafios para a operacionalização e qualificação do acolhimento, como: ausência de articulação em redes integradas, excesso de demandas de atendimento, ausência de capacitação e de espaços democráticos e reflexivos para organização do processo de trabalho em saúde ${ }^{13}$. Um serviço pode ser acessível quanto ao horário de funcionamento e localização, mas possuir barreiras organizacionais, como, por exemplo, baixa implantação do acolhimento ou baixa qualidade desse acolhimento, tanto por carência de profissionais capacitados para a classificação de risco e vulnerabilidade quanto pela lógica de organização desse acolhimento.

Barreiras organizacionais para o acesso constituem-se em problema ainda presente 
nas UBS brasileiras, inclusive a partir da percepção dos usuários. O modelo tradicional de organização da recepção, com fichas e filas, gera, na experiência de quem usa os serviços de $A B$, a disputa de vagas de atendimento e o incentivo a 'dormir na fila' para tentar o acesso à consulta ${ }^{14}$. É preciso repensar o modelo tradicional de organização do acesso nas UBS brasileiras, se o objetivo for a ampliação do acesso e da resolutividade na $\mathrm{AB}$, por exemplo, com a adoção de ferramentas, como telefone e internet, para facilitar a marcação de consultas e potencializar o vínculo UBS-usuário.

Tabela 1. Primeiro Contato: indicadores por dimensão - comparação entre os ciclos 1 e 2 do PMAQ-AB, Brasil, 2012 e 2014

\begin{tabular}{|c|c|c|c|c|c|}
\hline \multirow{2}{*}{ Indicadores } & \multicolumn{2}{|c|}{ Brasil Ciclo 1} & \multicolumn{2}{|c|}{ Brasil Ciclo 2} & \multirow{2}{*}{ Valor de $\mathrm{p}$} \\
\hline & $\mathbf{n}$ & $\%$ & n & $\%$ & \\
\hline \multicolumn{6}{|l|}{ Horário de funcionamento } \\
\hline UBS funcionam 5 ou mais dias na semana ${ }^{1}$ & 12050 & 93,7 & 12653 & 97,8 & $<0,001$ \\
\hline UBS funcionam 8 horas/dia ou mais ${ }^{1}$ & 11438 & 88,9 & 10557 & 81,6 & $<0,001$ \\
\hline \multicolumn{6}{|l|}{ Acolhimento e Agendamento de consultas na $A B$} \\
\hline Acolhimento implantado na UBS2 & 12690 & 80,9 & 15299 & 97,6 & $<0,001$ \\
\hline $\begin{array}{l}\text { Caso o usuário tenha um problema que não seja } \\
\text { recomendável o agendamento para outro dia, há } \\
\text { reserva de vagas para atendimento no mesmo dia² }\end{array}$ & 10177 & 64,9 & 13800 & 88,1 & $<0,001$ \\
\hline $\begin{array}{l}\text { Usuários podem marcar consultas todos os dias da } \\
\text { semana }{ }^{3}\end{array}$ & 32283 & 55,2 & 28081 & 47,0 & $<0,001$ \\
\hline $\begin{array}{l}\text { Para marcar consulta, usuários vão à UBS e, para pe- } \\
\text { gar ficha, é necessário fazer fila antes da UBS abrir³ }\end{array}$ & 17879 & 30,6 & 14583 & 24,4 & $<0,001$ \\
\hline \multicolumn{6}{|c|}{$\begin{array}{l}\text { Fontes: Banco PMAQ-AB, ciclo 1, 2012. Disponível em: http://dab.saude.gov.br/portaldab/ape_pmaq.php?conteudo=microdados. Banco } \\
\text { PMAQ-AB, ciclo 2, 2014. Disponível em: http://dab.saude.gov.br/portaldab/ape_pmaq.php?conteudo=2_ciclo. }\end{array}$} \\
\hline \multicolumn{6}{|l|}{ Variável que admite múltiplas respostas. } \\
\hline \multicolumn{6}{|l|}{ 1: N de UBS: 12.860; } \\
\hline \multicolumn{6}{|l|}{ 2: N de EqSF: 15.668; } \\
\hline$N$ de usuários: & & & & & \\
\hline
\end{tabular}

Na tabela 2, são apresentados os indicadores PMAQ-AB de longitudinalidade, nas dimensões continuidade da relação profissional-paciente e qualidade da relação profissional-paciente (vínculo).

Na continuidade da relação profissional-paciente, observaram-se resultados inferiores no ciclo 2 para a maior parte das variáveis. O único resultado superior encontrado no ciclo 2 foi o usuário ser sempre atendido pelo mesmo enfermeiro na UBS, de $57,9 \%$ para $61,6 \%$ (tabela 2 ). A continuidade da relação profissional-paciente mostrou baixos resultados em ambos os ciclos. Os indicadores de vínculo apresentaram leve melhora principalmente quanto ao usuário sentir-se à vontade em conversar com a equipe, contudo, ainda demandando preocupação.

Para a qualidade da relação profissional-paciente (vínculo), observaram-se resultados superiores no ciclo 2 nos indicadores sobre tempo de consulta e no indicador 'Usuário se sente à vontade para falar com a equipe sobre suas preocupações, problemas sociais e familiares'. Todavia, é importante observar que, em ambos os ciclos, apenas 63\% dos usuários conseguem tirar dúvidas após as consultas; e cerca de $60 \%$ se sentem à vontade para falar sobre suas questões, 
resultados que ainda demandam de melhorias para o fortalecimento do vínculo com o serviço de saúde.

Em síntese, para o atributo longitudinalidade, os resultados são, em geral, insatisfatórios, especialmente para a continuidade da relação profissional-paciente, mas também inadequados para a qualidade da relação profissional-paciente. Os resultados mais negativos se referiram ao tempo de atuação do profissional na equipe e à busca ativa dos profissionais no caso de abandono de tratamento. No entanto, o tempo das consultas (médico e enfermeiro) para o usuário falar sobre suas preocupações apresentou resultados satisfatórios. Melhorias são necessárias para a efetivação da longitudinalidade, que variam desde fatores da organização do serviço - como busca ativa, visita domiciliar de puericultura e construção da relação profissional-paciente, resultando em acessibilidade ao profissional após a consulta - até questões mais abrangentes - como a fixação de profissionais e desprecarização das relações de trabalho.

Uma gama de estudos tem associado a continuidade do cuidado e APS como fonte regular de cuidados a resultados positivos mais amplos, como a diminuição de internações e maior satisfação do paciente ${ }^{15-17}$.

Dois elementos principais que constituem a qualidade da assistência, o técnico e o interpessoal, estão inter-relacionados. Uma melhor comunicação e relação entre médicos e pacientes favoreceria a qualidade técnica da atenção prestada ao usuário ${ }^{18}$. A confiança no médico aumenta se o acompanhamento do usuário for feito pelo mesmo profissional de saúde ao longo do tempo, assim como a adesão do tratamento é fortalecida ${ }^{19}$. Essas são expressões do atributo vínculo longitudinal nos serviços de APS20.

Outro ponto relevante é que um maior tempo de consulta está relacionado com uma melhor qualidade do atendimento, envolvendo melhor explicação do problema e das solicitações diagnósticas e maior preocupação do médico sobre a compreensão do paciente ${ }^{21}$.

A seleção de parte dos indicadores da tabela 2 se baseia na evidência que a longitudinalidade envolve pré-condições como uma oferta adequada de serviços de APS e mecanismos de fixação de profissionais ${ }^{20,22}$. Entende-se que uma menor rotatividade dos profissionais e maior tempo de atuação na equipe pode indicar maior possibilidade de relação interpessoal desses profissionais com os usuários. Contudo, um dos resultados contundentes demonstra que menos da metade dos profissionais respondentes do PMAQ-AB estão nas equipes três anos ou mais.

A rotatividade profissional na ESF pode estar associada com diferentes aspectos. Alguns deles são: quanto mais tempo de formado, menor a rotatividade profissional; quanto maior a distância da UBS do local de moradia do profissional, maior a rotatividade; quanto menor a percepção de capacitação para o serviço, maior a rotatividade de profissionais ${ }^{23}$. São aspectos da $A B$ que influenciam de maneira positiva a continuidade da relação profissional-paciente e que estão relacionados com a maior satisfação do profissional no desempenho do trabalho.

Estudo sobre a relação do Programa Mais Médicos (PMM) e PCATool nas cinco regiões do Brasil identificou que os escores em todos os atributos foram favoráveis, com exceção do primeiro contato. $\mathrm{O}$ autor discute que uma importante contribuição para tal resultado foi a formação em medicina de família e comunidade em $100 \%$ dos médicos cubanos entrevistados, além da média de 5 anos de experiência de atuação ${ }^{24}$. Tal estudo retoma a importância e necessidade de avanços estruturais na APS, como formação na área, infraestrutura adequada e plano de carreira SUS. 
Tabela 2. Longitudinalidade: indicadores por dimensão - comparação entre os ciclos 1 e 2 do PMAQ-AB, Brasil, 2012 e 2014

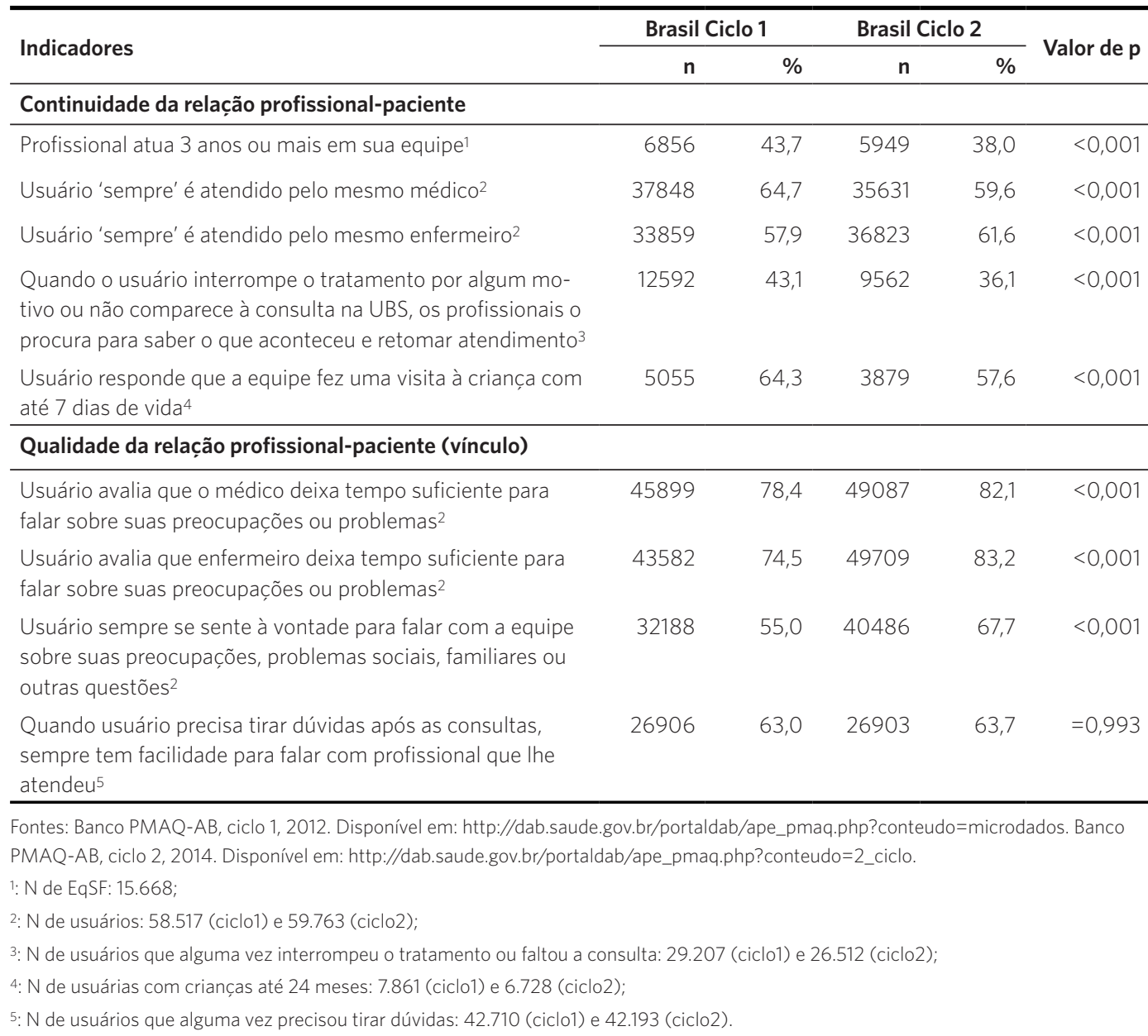

Na tabela 3, são apresentados os indicadores selecionados para aferir a abrangência/ integralidade, nas dimensões serviços realizados/escopo de ações e resolutividade.

Em relação ao escopo de ações, observam-se alguns resultados superiores no ciclo 2 , que se referem às consultas de médicos, realização pelas usuárias de pré-natal na UBS (de 70,6\% para $77,2 \%)$ e realização do procedimento sutura de ferimentos. Contudo, percebem-se valores inferiores no ciclo 2 quanto à realização pelos usuários da maioria de consultas de hipertensão e diabetes na UBS.

A resolutividade, relacionada com a ampliação do apoio às equipes por Núcleo de Apoio à Saúde da Família (Nasf) (de 56,1\% para 61,0\%) e Centro de Atenção Psicossocial
(Caps) (de $47,7 \%$ para $63,8 \%$ ), foi fortalecida, contudo, ainda insuficiente para todo o conjunto de respostas que a APS precisa oferecer ante a complexidade dos processos saúde-doença.

$\mathrm{O}$ apoio matricial e do Caps tem-se estruturado aos poucos no Brasil, com melhorias na qualidade da $\mathrm{AB}^{25}$. Desde sua implantação, são observados os avanços do Nasf por meio de ações como educação permanente, construção conjunta, ações clínicas compartilhadas, discussões de processo de trabalho e intervenções no território. Entretanto, extremamente desiguais de acordo com as regiões do País ${ }^{\mathbf{2 4}}$.

A ampliação do escopo de ações realizadas (consultas e procedimentos) foi paralela 
à redução no acompanhamento a programas específicos, aqui exemplificado pelo hiperdia. É importante destacar que a experiência dos usuários foi menos valorizada no instrumento do ciclo 2, fator que precisaria de uma revisão para o próximo ciclo. $\mathrm{O}$ apoio do Nasf e Caps às equipes, apesar de melhorado, contempla pouco mais da metade das equipes. $\mathrm{Ou}$ seja, as unidades de atenção básica necessitam ampliar seu escopo de atuação, disponibilizando mais serviços não apenas para atender às necessidades de saúde da população, mas também para qualificar o acompanhamento de grupos prioritários, em especial, o cuidado das pessoas com doenças crônicas.

Tendo em conta os diferentes sentidos da integralidade, é importante destacar que, para a análise do atributo abrangência/integralidade, foram selecionadas apenas condições objetivas de organização do serviço e percepção dos usuários ${ }^{3}$. Não se extrapolou para outras noções de integralidade como, por exemplo, uma abordagem integral do indivíduo ${ }^{26}$; ou a integração dos serviços por meio de redes assistenciais ${ }^{27}$.

Estudo sobre experiências dos usuários apontou, como sugestões feitas pelos usuários: a ampliação da oferta de profissionais e serviços, a maior disponibilidade de medicamentos, a ampliação da oferta de exames, implantação do serviço de urgência/emergência e aumento de realização de visitas domiciliares ${ }^{28}$.

A oferta de consultas odontológicas nas equipes de $\mathrm{AB}$ ocorre em menos de $80 \%$ das UBS, o que pode ser atribuído tanto à falta do profissional quanto à falta de equipamentos e insumos, mas, principalmente, ao fato de que existe uma relação (média) nacional de uma equipe de saúde bucal para cada duas EqSF, dificultando o acesso da população aos atendimentos odontológicos. Estudo com resultados específicos de saúde bucal nas UBS mostrou que menos da metade dos usuários entrevistados referiram conseguir marcar consulta com odontólogo nas UBS brasileiras ${ }^{29}$.

Nos grupos prioritários de cuidado continuado, como pessoas com hipertensão arterial sistêmica, diabetes mellitus e pré-natal, observou-se que cerca de $20 \%$ a $25 \%$ de usuários não fizeram as consultas na UBS nos últimos seis meses. Considerando o atributo de primeiro contato em que o horário de funcionamento atende às necessidades, talvez o que justifique esse resultado sejam obstáculos organizacionais e de infraestrutura.

No Brasil, a maior parte das UBS não dispõe de condições estruturais para realização das ações que se espera que a $\mathrm{AB}$ desenvolva. Mesmo para a realização de um conjunto de serviços mínimos - consultas médicas, de enfermagem e odontológicas, dispensação de medicamentos pela farmácia e vacinação -, as estruturas estão muito aquém do necessário ${ }^{30}$.

Outros pontos importantes de observação para analisar a integralidade seria a dispensação de medicamentos, realização de exames e de procedimentos, pontos não aprofundados nesse estudo. 
Tabela 3. Integralidade: indicadores por dimensão - comparação entre os ciclos 1 e 2 do PMAQ-AB, Brasil, 2012 e 2014

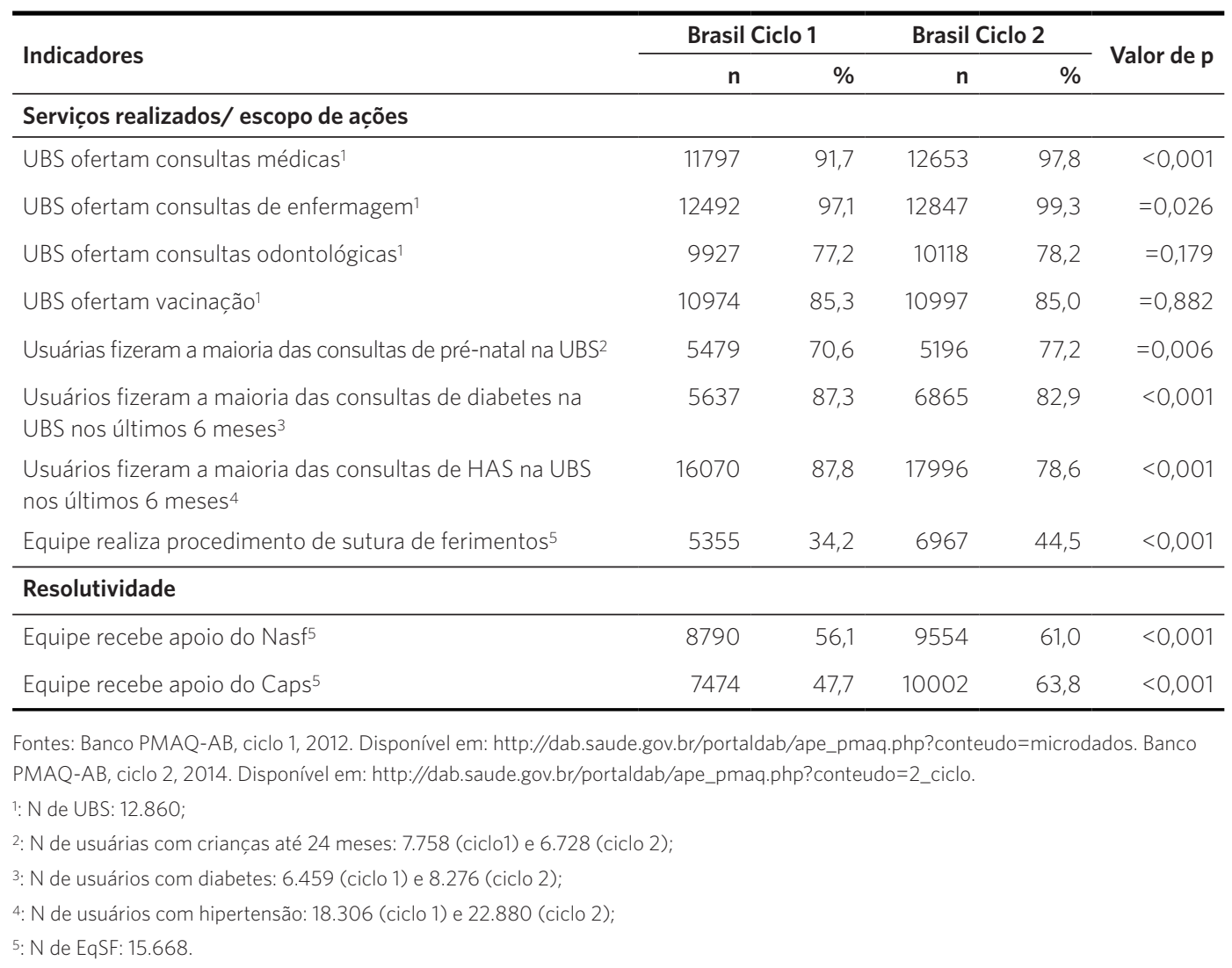

Na tabela 4, são apresentados os indicadores PMAQ-AB de coordenação, nas dimensões função de gatekeeper e acesso a serviços especializados; e continuidade informacional.

$\mathrm{Na}$ comparação entre os dois ciclos, os resultados foram diversificados. Na dimensão função de gatekeeper e acesso a serviços especializados, manteve-se a proporção de equipes que afirmaram que a consulta especializada é marcada pela UBS e depois informada ao usuário (cerca de 60\%), mas aumentou o número de equipes que afirmaram que os usuários já saem com a consulta agendada (26,4\% para $31,4 \%)$. Pelos resultados encontrados, houve aumento estatisticamente significativo em todas as formas de marcação (comparando ciclo 1 com ciclo 2). É nítida a necessidade de melhora na responsabilidade de marcação de consulta pela UBS, pois apenas cerca de metade dos usuários informou que a UBS marca consulta especializada. Negativamente, fica a observação do aumento de usuários que recebem ficha para 'algum' (indeterminado) serviço especializado (3,1\% para $13,7 \%)$. Vale destacar também que a referida pergunta admitia múltiplas respostas, e isso foi um fator que dificultou a análise.

Observou-se melhora na continuidade informacional: registros de usuários com hipertensão de maior risco (49,3\% para $60,3 \%)$ e na quantidade de equipes com implantação do prontuário eletrônico (11,5\% para 14,2\%), possivelmente relacionado pela indução do Ministério da Saúde da estratégia e-SUS AB nos municípios.

$\mathrm{O}$ atributo da coordenação apresentou os piores resultados entre os demais atributos, indicando dificuldades de acesso dos usuários às consultas especializadas, baixa integração 
entre os serviços da rede e necessidade de qualificação dos registros como mecanismo institucionalizado para coordenar esse acesso dos usuários aos serviços que necessitam. Um exemplo, tanto na perspectiva dos profissionais como dos usuários, são os baixos percentuais de UBS que se responsabilizam pela marcação de consultas especializadas.

Diferentes estudos sobre o tema, em especial, nas reformas pró-coordenação nos países europeus, mostram ser a coordenação uma preocupação crescente, assim como o avanço de medidas para melhorar a coordenação do cuidado em países com serviços nacionais de saúde com função de gatekeeper exercida pela APS ${ }^{31,1}$.É perceptível uma maior coordenação e integração da APS no sistema de saúde, em que os serviços ambulatoriais de primeiro contato estão integrados em um sistema de saúde de acesso universal e a atenção individual é garantida em todos os níveis de atenção à saúde ${ }^{32,33}$.

Já no caso brasileiro, diversos estudos encontraram deficiência na função de coordenação pela APS12,11,34-36.

Para que a $A B$ avance no sentido da oferta de cuidados continuados, é preciso investir nos mecanismos de integração e na melhoria da comunicação entre os serviços e entre profissionais da $\mathrm{AB}$ e da Atenção Especializada (AE). Estratégias de integração, tais como gestão de casos, gestão de lista de espera, revisão do prontuário, protocolos clínicos e sistema integrado de informação, são estratégias potentes de coordenação que podem contribuir para o fortalecimento da função de filtro da APS ${ }^{27}$. Ademais, é necessário articular a prática da APS no cenário regional, ganhando escala e potencialidades, como forma de garantir um sistema de saúde menos fragmentado.

A articulação entre serviços e ações para uma prestação de cuidado mais integral e sincronizada pode ser potencializada por estratégias de integração da rede e investimento em Tecnologias de Informação e Comunicação (TIC) ${ }^{37}$. A implantação das TIC é precária, assim como a continuidade informacional entre $\mathrm{AB}$ e $\mathrm{AE}$, por exemplo, por meio de prontuário eletrônico compartilhado, é muito baixa em todo o País.

Experiências isoladas nacionalmente, como a do município de Curitiba, mostram bons resultados para o atributo da coordenação do cuidado. Em relação ao sistema de informação, refletem os investimentos feitos pelo município no sistema informatizado, tanto para resultados de exames laboratoriais de análises clínicas disponibilizados on-line no prontuário como na operacionalização da marcação de consultas especializadas ${ }^{\mathbf{3 8}}$.

Cabe ressaltar a importância de profissionais qualificados também para exercer a função de filtro para o acesso aos serviços especializados. A qualificação dos profissionais pode evitar a burocratização excessiva (busca do profissional da $\mathrm{AB}$ somente para encaminhamentos) e o aumento (e excesso) do número de encaminhamentos aos serviços especializados ${ }^{39}$.

Problemas relacionados com a integração entre níveis - tais como a falta de integração e comunicação entre diferentes prestadores e a insuficiência de fluxos formais para atenção hospitalar - refletem a limitação da gestão municipal de, isoladamente, responder pelo conjunto de ações próprias da APS e a necessidade de articulação regional11,36. Ainda que alguns avanços para melhorar a integração da rede sejam observados - como estruturas regulatórias nas secretarias municipais e nas UBS com descentralização de funções para o nível local, organização dos fluxos, prontuários eletrônicos e ampliação da oferta de serviços especializados municipais -, são mecanismos insuficientes e que apenas minimizam os efeitos da fragmentação do sistema de saúde brasileiro.

Vale destacar que o atributo da coordenação foi um dos que possibilitaram menor comparabilidade entre ciclos 1 e 2, devido a mudanças no instrumento. Elementos centrais, como contato institucionalizado entre profissionais da rede de atenção e referências de encaminhamento, foram excluídos do novo instrumento. Algumas variáveis se dispersaram no instrumento do ciclo 2 para especificidades dos questionários da saúde bucal ou Nasf. 
Tabela 4. Coordenação: indicadores por dimensão - comparação entre os ciclos 1 e 2 do PMAQ-AB, Brasil, 2012 e 2014

\begin{tabular}{|c|c|c|c|c|c|}
\hline \multirow{2}{*}{ Indicadores } & \multicolumn{2}{|c|}{ Brasil Ciclo 1} & \multicolumn{2}{|c|}{ Brasil Ciclo 2} & \multirow{2}{*}{ Valor de $\mathrm{p}$} \\
\hline & $\mathbf{n}$ & $\%$ & $\mathrm{n}$ & $\%$ & \\
\hline \multicolumn{6}{|l|}{ Função de gatekeeper e acesso a serviços especializados } \\
\hline \multicolumn{6}{|l|}{$\begin{array}{l}\text { Quando o usuário necessita ser encaminhado para consulta espe- } \\
\text { cializada*: }\end{array}$} \\
\hline A consulta é marcada pela UBS e depois informada ao usuário & 9661 & 61,6 & 9442 & 60,3 & $p=0.1147$ \\
\hline O usuário já sai da UBS com a consulta agendada & 4136 & 26,4 & 4918 & 31,4 & $p<0.0001$ \\
\hline$N E q A B$ & 15668 & & & & \\
\hline \multicolumn{6}{|l|}{$\begin{array}{l}\text { Usuários quando são atendidos na UBS e necessitam ser encami- } \\
\text { nhados para outros profissionais ou especialistas*: }\end{array}$} \\
\hline Saem da UBS com a consulta agendada & 5479 & 12,9 & 5830 & 16,9 & $p<0.0001$ \\
\hline A consulta é marcada pela UBS e depois informada ao usuário & 19738 & 46,5 & 15878 & 46,0 & $p<0.0001$ \\
\hline A consulta é marcada por usuário na central de marcação & 5976 & 14,1 & 5606 & 16,2 & $p<0.0001$ \\
\hline $\begin{array}{l}\text { Recebem ficha de referência para profissional/serviço especiali- } \\
\text { zado determinado }\end{array}$ & 7815 & 18,4 & 9290 & 26,9 & $p<0.0001$ \\
\hline $\begin{array}{l}\text { Recebem ficha de referência para algum profissional/serviço } \\
\text { especializado }\end{array}$ & 1308 & 3,1 & 4717 & 13,7 & $p<0.0001$ \\
\hline N usuários que alguma vez precisou marcar consultas com especialistas & 42461 & & 34506 & & \\
\hline Usuárias gestantes foram orientadas sobre a maternidade do parto & 3699 & 67,5 & 3080 & 59,3 & $p<0.0001$ \\
\hline N usuárias com crianças até 24 meses & 5479 & & 5196 & & \\
\hline \multicolumn{6}{|l|}{ Continuidade informacional } \\
\hline Possui registro das pessoas com HAS com maior risco/gravidade & 7721 & 49,3 & 9442 & 60,3 & $p<0.0001$ \\
\hline EqAB possui prontuário eletrônico implantado & 2267 & 14,5 & 2665 & 17,0 & $p<0.0001$ \\
\hline $\begin{array}{l}\text { EqAB possuem prontuário eletrônico e ele está integrado com } \\
\text { outros pontos da rede de atenção }\end{array}$ & 1800 & 11,5 & 2229 & 14,2 & $p<0.0001$ \\
\hline$N E q A B$ & 15668 & & & & \\
\hline
\end{tabular}

Fontes: Banco PMAQ-AB, ciclo 1, 2012. Disponível em: http://dab.saude.gov.br/portaldab/ape_pmaq.php?conteudo=microdados. Banco PMAQ-AB, ciclo 2, 2014. Disponível em: http://dab.saude.gov.br/portaldab/ape_pmaq.php?conteudo=2_ciclo.

*Variável que admite múltiplas respostas.

\section{Considerações finais}

O conjunto de análises realizadas com base nos bancos de dados do PMAQ permite um panorama nacional da situação atual do acesso e qualidade da APS no País. Os atributos analisados partiram do pressuposto dos benefícios que o modelo de atenção à saúde com base na APS proporciona à população, e à organização do sistema de saúde, tendo a APS a função de porta de entrada e coordenadora do cuidado.
O presente artigo demonstrou a importância de medir os atributos da APS, mesmo em um espaço curto de tempo, como forma de qualificar os serviços e, consequentemente, o atendimento aos usuários; demonstrando que os estudos de avaliação dos serviços de saúde podem ser úteis para fornecer caminhos e parâmetros para a tomada de decisão sobre a política governamental.

É importante considerar o alinhamento do PMAQ-AB a uma série de outras iniciativas 
nacionais do Ministério da Saúde, como PMM, Programa Requalifica UBS, e-SUS AB etc. No momento da avaliação externa do PMAQ ciclo 1, o provimento pelo PMM ainda não fora implantado; já no ciclo 2, o PMM estava em fase inicial. Em outras palavras, seriam necessários outros estudos e maior tempo de existência dos programas para avaliar o impacto dessas iniciativas na qualidade da APS. Apesar de alguns resultados positivos obtidos entre um ciclo e outro do PMAQ-AB, não se pretende atribuir o impacto dos resultados exclusivamente ao Programa, visto que um estudo dessa envergadura demandaria outra metodologia e aprofundamento. É necessário, também, atentar para o curto espaço de tempo entre os dois ciclos do PMAQ-AB, que dificulta intervenções para enfrentar os problemas identificados.

$\mathrm{Na}$ comparação entre os resultados dos ciclos 1 e 2 do PMAQ-AB, observou-se alguma melhoria da qualidade nas equipes participantes. Os atributos que apresentaram melhores resultados do PMAQ-AB foram os de primeiro contato e integralidade, com alguns indicadores que se mostraram superiores no ciclo 2. Tal resultado confirma a progressiva constituição da UBS como serviço de porta de entrada acessível, com escopo diversificado de ações ofertadas, ainda que insuficientes. Os atributos com pior avaliação foram a longitudinalidade com baixa continuidade e qualidade da relação profissional-paciente e a coordenação, que recebeu a pior avaliação por dificuldades de acesso dos usuários às consultas especializadas e insuficiente registros. Outro ponto que merece atenção é uma redução no acompanhamento dos programas de saúde, aqui representados pelas variáveis visita de puericultura, indicação de maternidade e consultas a pessoas com hipertensão arterial e diabetes, o que pode estar relacionado com a redução do tempo de vínculo dos profissionais. Deve-se lembrar que não há informação disponível que permita conhecer se e quando projetos de intervenção foram implementados como previu a fase 3 do PMAQ-AB.

Os resultados aqui discutidos podem nos remeter a algumas observações:

- a importância da linearidade entre os instrumentos dos ciclos do PMAQ-AB a fim de melhor avaliar a evolução das equipes entrevistadas;

- a possibilidade, mediante variáveis do PMAQ-AB, de visualizar o funcionamento da $A B$ em relação aos quatro atributos essenciais da APS: primeiro contato, longitudinalidade, abrangência/integralidade e coordenação;

- a necessidade de estudos que façam uma avaliação do PMAQ-AB associando outros programas governamentais de qualificação da APS.

É importante destacar que um sistema de saúde com ênfase na APS deve estar acompanhado da capacidade de medir o grau de orientação dessa APS. Logo, reafirma-se a necessidade de manutenção de uma avaliação contínua e processual da atenção básica, como forma de medir sua trajetória ao longo do tempo e identificar quais são os aspectos que demandam maior investimento. Para tal, manter o PMAQ-AB e a coerência nos seus instrumentos no decorrer dos ciclos auxiliaria nesse comparativo, mostrando-se um importante instrumento rumo à garantia da qualidade e maior resolutividade da APS, objetivo central para um sistema de saúde universal, equânime e integral. 


\section{Referências}

1. Starfield B, Shi L, Macincko J. Contribution of Primary care to health systems and health. Milbank Q [internet]. 2005 [acesso em 2018 maio 5]; 83(3):457502. Disponível em: http://www.ncbi.nlm.nih.gov/ pmc/articles/PMC2690145/.

2. Rifkin SB, Walt G. Why health improves: defining the issues concerning 'comprehensive primary health care and selective primary health care'. Soc Sci Med. [internet]. 1986 [acesso em 2018 abr 28]; 23(6):559-566. Disponível em http://www.ncbi. nlm.nih.gov/pubmed/3764507.

3. Starfield B. Atenção Primária: equilíbrio entre necessidades de saúde, serviços e tecnologia. Brasília, DF: Unesco: Ministério da Saúde; 2002.

4. Brasil. Ministério da Saúde. Secretaria de Atenção à Saúde. Portaria 2.488, de 21 de outubro de 2011. Aprova a Política Nacional de Atenção Básica, estabelecendo a revisão de diretrizes e normas para a organização da Atenção Básica, para a Estratégia Saúde da Família (ESF) e o Programa de Agentes Comunitários de Saúde (PACS). Brasília, DF: Ministério da Saúde, Diário Oficial da União. 22 Out 2011.

5. Almeida PF, Giovanella L. Avaliação em Atenção Básica à Saúde no Brasil: mapeamento e análise das pesquisas realizadas e/ou financiadas pelo Ministério da Saúde entre os anos de 2000 e 2006. Cad. Saúde Pública. 2008; 24(8):1727-1742.

6. Brasil. Ministério da Saúde. Secretaria de Atenção à Saúde. Portaria 1.654, de 19 de julho de 2011. Institui, no âmbito do Sistema Único de Saúde, o Programa Nacional de Melhoria do Acesso e da Qualidade da Atenção Básica (PMAQ-AB) e o Incentivo Financeiro do PMAQ-AB, denominado Componente de Qualidade do Piso de Atenção Básica Variável - PAB Variável. Diário Oficial da União. 19 Jul 2011.

7. Trindade TG. Associação entre a extensão dos atributos de atenção primária e qualidade do manejo da hipertensão arterial em adultos adscritos à rede de atenção primária à saúde de Porto Alegre [disserta- ção] [internet]. Porto Alegre: Universidade Federal do Rio Grande do Sul; 2007. 145p. [acesso em 2018 maio 15]. Disponível em: https://www.lume.ufrgs.br/bitstream/handle/10183/12947/000636050. pdf? sequence $=1$.

8. Gonçalves MR. A qualidade da atenção primária e o manejo do diabetes mellitus. RBMFC. 2013; 8(29):235-243.

9. Giovanella L, Mendonça MH. Atenção primária à saúde. In: Giovanella L, Escorel S, Lobato LVC, et al. organizadores. Políticas e Sistema de Saúde no Brasil. Rio de Janeiro: Fiocruz; 2012. p. 493-546.

10. Macincko J, Starfield B, Shi L. The contribution of primary care systems to health outcomes within Organization for Economic Cooperation and Development (OECD) countries, 1970-1998. Health Serv Res. [internet]. 2003 [acesso em 2018 abr 15]; 38(3):831-865. Disponível em: http://www.jhsph. edu/sebin/k/k/2003_HSR_Macinko.pdf.

11. Almeida PF, Giovanella L, Mendonça MHM, et al. Desafios à coordenação dos cuidados em saúde: estratégias de integração entre níveis assistenciais em grandes centros urbanos. Cad. Saúde Pública. 2010; 26(2):286-298.

12. Escorel S, Giovanella L, Mendonça MHM, et al. O Programa de Saúde da Família e a construção de um novo modelo para a atenção básica no Brasil. Revista Pan-americana de Salud Publica. 2007; 21(2):164-176.

13. Mitre SM, Andrade EIG, Cotta RMM. Avanços e desafios do acolhimento na operacionalização e qualificação do Sistema Único de Saúde na Atenção Primária: um resgate da produção bibliográfica do Brasil. Ciênc Saúde Colet.; 2012, 17(8):2071-2085.

14. Souza ECF, Vilar RLA, Rocha NSPD, et al. Acesso e acolhimento na atenção básica: uma análise da percepção dos usuários e profissionais de saúde. Cad. Saúde Pública, Rio de Janeiro, 2008; 24(supl.1):s100-s110. 
15. Kringos DS, Boerma WGW, Hutchinson A, et al. The breadth of primary care: a systematic literature review of its core dimensions. BMC Health Services Research [internet]. 2010 [acesso em 2018 mar 9]; p. 10-65. Disponível em: http://bmchealthservres.biomedcentral.com/ articles/10.1186/1472-6963-10-65.

16. Cunha EM, Giovanella L. Longitudinalidade/continuidade do cuidado: identificando dimensões e variáveis para a avaliação da Atenção Primária no contexto do sistema público de saúde brasileiro. Ciênc Saúde Colet. 201; 16(Supl.1):1029-1042.

17. Damasceno SS. Avaliação da Orientação à Atenção Primária à Saúde da Criança [dissertação]. João Pessoa: Universidade Federal da Paraíba; 2014. 140 p.

18. Donabedian A. La calidad de la asistencia. ¿̨Cómo podría ser evaluada? Rev Calidad Asistencial [internet]. 2001 [acesso em 2018 mar 29]; 16(1):580-587. Disponível em: http://www.fadq.org/Portals/0/Investigacion/ Monografico\% 20Avedis\%202parte.pdf.

19. Mainous AG, Baker R, Love MM, et al. Continuity of care and trust in one's physician: evidence from Primary Care in the United States and United Kingdom. Family Medicine [internet]. 2001 [acesso em 2018 abril 10]; 33(1):22-27. Disponível em: http:// www.stfm.org/Portals/49/Documents/FMPDF/ FamilyMedicineVol33IssuelArch22.pdf.

20. Cunha EM. Vínculo Longitudinal na Atenção Primária: avaliando os modelos assistenciais do SUS [tese]. [Rio de Janeiro]: Fundação Oswaldo Cruz; 2009. 150p.

21. Caprara A, Rodrigues J. A relação assimétrica médico-paciente: repensando o vínculo terapêutico. Ciênc Saúde Colet. 2004; 9(1):139-146.

22. Campos RTO, Ferrer AL, Gama CAP, et al. Avaliação da qualidade do acesso na atenção primária de uma grande cidade brasileira na perspectiva dos usuários. Saúde debate. 2014; 38(esp):252-264.

23. Campos VA, Malik AM. Satisfação no trabalho e rotatividade dos médicos do Programa de Saúde da
Família. Rev. Adm. Pública. 2008; 42(2):347-68.

24. Kemper ES. Programa Mais Médicos: contribuições para o fortalecimento da Atenção Primária à saúde [tese]. [Brasília, DF]: Universidade de Brasília; 2016.

25. Fonseca Sobrinho D, Machado ATGM, Lima AMLD, et al. Compreendendo o apoio matricial e o resultado da certificação de qualidade nas áreas de atenção à criança, mulher, diabetes/hipertensão e saúde mental. Saúde debate. 2014; 38(esp):83-93.

26. Mattos RA. Os sentidos da integralidade: algumas reflexões acerca de valores que merecem ser defendidos. In: Mattos RA, organizador. Os sentidos da integralidade na atenção e no cuidado à saúde. Rio de Janeiro: IMS/UERJ-ABRASCO; 2001. p. 39-66.

27. Mendes EV. As redes de atenção à saúde [internet]. Brasília, DF: OPAS; 2011. [acesso em 2018 jun 1]. Disponível em: https://www.paho.org/bra/index. php?option=com $\_$docman\&view=download\&category slug=servicos-saude-095 $\&$ alias $=1402$-as-redes -atencao-a-saude-2a-edicao-2\&Itemid $=965$.

28. Brandão ALRBS, Giovanella L, Campos CEA. Avaliação da atenção básica pela perspectiva dos usuários: adaptação do instrumento EUROPEP para grandes centros urbanos brasileiros. Ciênc Saúde Colet. 2013; 18(1):103-114.

29. Casotti E, Contarato PC, Fonseca ABM, et al. Atenção em Saúde Bucal no Brasil: uma análise a partir da Avaliação Externa do PMAQ-AB. Saúde debate. 2014; 38(esp):140-157.

30. Bousquat A, Giovanella L, Fausto MCR, et al. Tipologia das Unidades Básicas de Saúde Brasileiras: os 5R. Cad. Saúde Pública. 2017; 33(8):e00037316.

31. Giovanella L, Stegmüller K. Tendências de reformas na atenção primária à saúde em países europeus. JMPHC [internet]. 2014 [acesso em 2018 maio 05]; 5(1):101-113. Disponível em: http://www.jmphc. com/ojs/index.php/01/article/view/179.

32. Giovanella L. A atenção primária à saúde nos países 
da União Européia: configurações e reformas organizacionais na década de 1990. Cad. Saúde Pública. 2006; 22(5):951-963.

33. Davis K, Stremikis K, Schoen C, et al. Mirror, mirror on the wall. How the Performance of the U.S. Health Care System Compares Internationally. The Commonwealth Fund; 2014. [acesso em 2018 out 9]. Disponível em: https://www.commonwealthfund. org/sites/default/files/documents/__media_files_ publications_fund_report_2014_jun_1755_davis_ mirror_mirror_2014.pdf.

34. Ibañez N, Rocha JSY, Castro PC, et al. Avaliação do desempenho da atenção básica no estado de São Paulo. Ciênc Saúde Colet. 2006;11(3):683-703.

35. Harzhein E. Reforma da Atenção Primária à Saúde na cidade do Rio de Janeiro - avaliação dos três anos de Clínicas da Família. Pesquisa avaliativa sobre aspectos de implantação, estrutura, processo e resultados das Clínicas da Família na cidade do Rio de Janeiro. Porto Alegre: OPAS; 2013.

36. Fausto MCR, Giovanella L, Mendonça MHM, et al. A posição da Estratégia Saúde da Família na rede de atenção à saúde na perspectiva das equipes e usuários participantes do PMAQ-AB 2012. Saúde debate [internet]. 2014 [acesso em 2018 mar 20], 38(esp):13-33. Disponível em http://www.scielo.br/ pdf/sdeb/v38nspe/0103-1104-sdeb-38-spe-0013.

pdf.

37. Almeida PF, Santos AM. Diálogos em busca da coordenação do cuidado: linha de chegada ou novo itinerário? In: Almeida PF, Santos AM. Souza MKB, organizadores. Atenção primária à saúde na coordenação do cuidado em regiões de saúde. Salvador: EDUFBA; 2015. p. 277-303.

38. Chomatas E, Vigo A, Marty I, et al. Avaliação da presença e extensão dos atributos da atenção primária em Curitiba. Revista Brasileira de Medicina de Família e Comunidade [internet]. 2013 [acesso em 2018 abril 4], 8(29):294-303. Disponível em: http:// www.rbmfc.org.br/rbmfc/article/view/828/587.

39. Leao CDA, Caldeira AP, Oliveira MMC. Atributos da atenção primária na assistência à saúde da criança: avaliação dos cuidadores. Rev. Bras. Saude Mater. Infant. 2011 set; 11(3):323-334.

\footnotetext{
Recebido em 10/06/2018

Aprovado em 30/08/2018

Conflito de interesses: inexistente

Suporte financeiro: não houve
} 